\title{
Association between Cold Face Test-induced vagal inhibition and cortisol response to acute stress
}

\author{
ROBERTO LA MARCA, ${ }^{\mathrm{a}}$ PATRICIA WALDVOGEL,${ }^{\mathrm{a}}$ HANNA THÖRN,${ }^{\mathrm{a}}$ MÉLANIE TRIPOD,${ }^{\mathrm{a}}$ \\ PETRA H. WIRTZ, ${ }^{\mathrm{a}}$ JENS C. PRUESSNER, ${ }^{\mathrm{b}}$ AND ULRIKE EHLERT ${ }^{\mathrm{a}}$ \\ ${ }^{a}$ Department of Clinical Psychology and Psychotherapy, University of Zurich, Zurich, Switzerland \\ ${ }^{\mathrm{b}}$ Departments of Psychiatry, Neurology, and Neurosurgery, McGill University \& Douglas Hospital Research Centre, Montréal, Québec, Canada
}

\begin{abstract}
Low vagal function is related to several disorders. One possible underlying mechanism linking the vagus nerve and disorders is the HPA axis. Thirty-three healthy male subjects participated in a stress task, while heart rate (HR), respiratory sinus arrhythmia (RSA), salivary cortisol, and mood were assessed. Vagal function was determined using baseline, stress-induced inhibition, and Cold Face Test (CFT)-induced stimulation. The stress task induced a significant increase in cortisol and HR, a decrease in RSA, and a worsening of mood. A linear regression model with the time from CFT onset until maximum bradycardia as the independent variable explained $17.9 \%$ of the total variance in cortisol in response to the stressor (mood: $36.5 \%$ ). The results indicate that a faster CFT response is associated with reduced cortisol increase and enhanced mood after acute stress. Our data support an inverse relationship between vagal function and the HPA axis.
\end{abstract}

Descriptors: Vagal function, Acute psychosocial stress, Cortisol reactivity

The vagus nerve $(\mathrm{VN})$ is the major constituent of the parasympathetic nervous system (PSNS), and innervates several vital organs. Functionally, the VN promotes rest and digest, while the sympathetic nervous system (SNS) promotes fight or flight. Vagal activity can be indexed by variation in inter-beat intervals (heart rate variability, HRV; Task Force, 1996). Reduced activity and reactivity levels of HRV have frequently been reported to be associated with somatic and mental disorders and mortality (Thayer \& Brosschot, 2005; Thayer \& Lane, 2007), while invasive electrical stimulation of the $\mathrm{VN}$ is used for the treatment of certain disorders (Bernstein, Barkan, \& Hess, 2006; Hord, Evans, Mueed, Adamolekun, \& Naritoku, 2003; Merrill, Jonsson, Minthon, Ejnell, C-son Silander, et al., 2006; Rush, George, Sackeim, Marangell, Husain, et al., 2000).

However, the underlying mechanisms linking lower activity of the VN and somatic and mental disorders are not fully understood. The hypothalamic-pituitary-adrenal (HPA) axis, with dysregulations of its own also being related to several somatic and mental disorders (e.g., Christensen \& Kessing, 2001; Tsigos \& Chrousos, 1994), may play a mediating role. In fact, the VN is assumed to possess a regulating effect on the HPA axis (Thayer \& Sternberg,

We gratefully acknowledge the help of Susanne Fischer in conducting the experiments. This study was supported by a grant to the first author from the Forschungs- und Nachwuchsförderungskommission der Universität Zürich.

Address correspondence to: Ulrike Ehlert, Department of Clinical Psychology and Psychotherapy, University of Zurich, Binzmuehlestrasse 14/Box 26, CH-8050 Zurich, Switzerland. E-mail: u.ehlert@ psychologie.uzh.ch
2006). From a structural point of view, the central autonomic network (CAN; Benarroch, 1997) provides a framework for an association between the two systems. The CAN contains complex direct and indirect interconnections between several structures, such as the medullary vagal nuclei and the paraventricular nucleus of the hypothalamus, but also the prefrontal cortex (PFC) and the amygdala (Benarroch, 1997). The CAN modulates psychophysiological resources in emotion (Thayer \& Friedman, 2002), with the PFC and the VN providing negative feedback on sympathoexcitatory and HPA axis responses (Benarroch, 1997; Palkovits, 1999; Porges, 2001; Thayer \& Friedman, 2002; Thayer \& Lane, 2000; Thayer \& Sternberg, 2006). Since HRV indexes the inhibitory control of the PFC on the amygdala (Thayer \& Sternberg, 2006), and inhibition plays an important role for the ability to rapidly adapt to demands (Thayer \& Friedman, 2002), HRV has been proposed as an index for the ability to adapt to changing demands from the environment (Thayer, 2007).

While a structural association between the VN and the HPA axis, with an inverse relation between the function (activity and reactivity) of the two systems, can be assumed, non-invasive psychophysiological studies examining HRV and cortisol during different conditions show inconsistent results. Baseline levels of HRV and cortisol were shown to be unrelated to each other (Gunnar, Porter, Wolf, Rigatsu, \& Larson, 1995; Johnsen, Hansen, Sollers, Murison, \& Thayer, 2002). In contrast, baseline levels of HRV were reported to be related to cortisol stress responses, but results are contradictory, with evidence for both negative (Johnsen et al., 2002) and positive (Gunnar et al., 1995) associations. Similarly, studies investigating stress-induced vagal 
inhibition report inconsistent findings: HRV and cortisol in response to stress (social, psychological, physical, workday challenges) show negative (Doussard-Roosevelt, Montgomery, \& Proges, 2003; Thayer, Hall, Sollers, \& Fischer, 2006) as well as no significant associations (Altemus, Redwine, Leong, Frye, Porges, \& Carter, 2001; Cacioppo, Malarkey, Kiegolt-Glaser, Uchino, Sgoutas-Emch, et al., 1995; Gunnar et al., 1995; Heilman, Bal, Bazhenova, Sorokin, Perlman, Hanley, \& Porges, 2008). Notably, hitherto all studies investigating the VN and the HPA stress reactivity examine vagal function comprising baseline activity and/or stress-induced vagal inhibition. To the best of our knowledge, associations between acute vagal stimulation and HPA stress reactivity have not yet been investigated.

The main purpose of the present study was to examine the relation between vagal function (baseline, inhibition, stimulation) and cortisol measures (baseline, stimulation). To stimulate the VN, the cold face test (CFT; Khurana \& Wu, 2006) was conducted (i.e., CFT-induced vagal stimulation). To induce a stress response of the VN (i.e., stress-induced vagal inhibition) and the HPA axis, the Montreal Imaging Stress Task (MIST, Dedovic, Renwick, Khalili-Mahani, Engert, Lupien, \& Pruessner, 2005) was applied. To our knowledge, this is the first study to examine the effects of the MIST on the PSNS, representing the second purpose of the present study.

\section{Methods and Materials}

\section{Study Participants}

Subjects were recruited by advertisement at the universities of Zurich. Inclusion criteria included male sex and an age range of 18 to 40 years and right-handedness. Exclusion criteria included depression, self-reported acute and chronic somatic or psychiatric disorders, medication in the last 2 months, the consumption of psychoactive substances, and excessive consumption of alcohol ( $>2$ alcohol beverages/day) or tobacco ( $>5$ cigarettes/day). Inclusion was restricted to male subjects, primarily due to gender differences in possible pain sensation during the CFT (Girdler, Maixner, Naftel, Stewart, Moretz, \& Light, 2005), but also due to the effect of oral contraceptives and menstrual cycle phase on cortisol and HRV (Kirschbaum, Kudielka, Gaab, Schommer, \& Hellhammer, 1999; Sato, Myake, Akatsu, \& Kumashiro, 1995). Participants received monetary compensation for their participation. The study was conducted in accordance with the Declaration of Helsinki and was approved by the cantonal ethics committee. Subjects provided written informed consent prior to participation.

\section{Procedure}

After arriving at the laboratory, subjects were fitted with the cardiorespiratory ambulatory device, and were seated in a comfortable chair. All tests were conducted in the same room at a constant temperature $\left(21^{\circ} \mathrm{C}\right)$, while subjects were sitting in front of a table with a computer and several available magazines. After a rest period of $30 \mathrm{~min}$, subjects were asked to fill out state mood questionnaires. This was followed by the MIST. Subjects were exposed in random order to the stress (MIST-S) and control condition (MIST-C), on two separate occasions 2 weeks apart. Mood questionnaires were again handed out after the termination of the task. To include cortisol stress recovery, the examination lasted for an additional $60 \mathrm{~min}$. At the end of the control examination, the CFT was conducted, while at the end of the stress examination, subjects were debriefed and informed about the cover story (examination of interaction between cognitive skills and physiological markers). Subjects who first participated in the stress condition were reassured that, on the next occasion, a cognitive but not a stress task would take place. This procedure was chosen to ensure that, at the beginning of each examination, all subjects, independent of the condition order, would expect a cognitive task to be conducted. With this procedure, we intended to avoid an anticipatory stress response in subjects who participated in the control condition in the second examination. At the end of the second examination, participants gave a second written informed consent allowing the further use of their data.

\section{Interventions}

Stress Task

To induce a multidimensional stress response, a slightly modified version of the MIST (Dedovic et al., 2005) was used, since the task was originally developed for fMRI environments. The MIST is a standardized computerized stress task combining challenging arithmetic problems with social-evaluative threat and can be carried out with (stress condition; MIST-S) or without time pressure and social evaluation (control condition; MIST-C). During the MIST-S, the program adapts the difficulty and time provided to solve the problems impeding a good performance ( $45 \%$ to $50 \%$ of correct answers). During the MIST-C, the difficulty of the arithmetic problems is randomly chosen, and neither time pressure nor social evaluation is applied. In both conditions, three blocks of 4 min each were run, with feedback of 2-3 min provided by the examiner in between blocks. The first feedback of the MIST-S consisted of informing the participants that their performance was poor, with the examiner inquiring whether the subjects were experiencing any application problems (e.g., problems with the keyboard). A fictitious study leader then informed the examiner by telephone to repeat the test. In the second feedback of the MIST-S, the study leader entered the examination room and interrogated the participants about individual problems (e.g., about school performance). Participants were then informed about the high costs due to a possible exclusion if they did not achieve a better performance. The last block was then started, while the study leader remained in the examination room during the first $3.5 \mathrm{~min}$. Before leaving the room, he instructed the examiner to continue with the normal procedure. At the very end of the stress examination, participants were debriefed. During the first and second feedback of the MIST-C, participants were provided with a neutral feedback. They were asked to perform a second and third block, respectively, in order to evaluate the time course of interactions between cognitive and physiological characteristics.

\section{Cold Face Test}

To extend the examination of vagal function, in addition to vagal baseline and stress-induced inhibitory measurement, the CFT was conducted in order to provoke vagal stimulation, since it mimics the diving reflex by inducing a trigeminal-vagal-mediated bradycardia (Arnold, 1999; Khurana, 2007; Khurana, Watabiki, Hebel, Toro, \& Nelson, 1980; Khurana \& Wu, 2006).

Bradycardia was induced by using a full-face mask (Dr. Winkler $\mathrm{GmbH}$, Ainring-Mitterfelden, Germany) covering wide parts of the face, with openings for the eyes avoiding an occulocardiac reflex, and for the nose and mouth allowing normal breathing. An additional cold pack (Nexcare, 3M Health Care, St. Paul, MN) was affixed to the full-face mask to augment the cold mass. While the room temperature was kept constant $\left(21^{\circ} \mathrm{C}\right)$, the temperature of 
the cold stimulus was $1{ }^{\circ} \mathrm{C}$. The sitting subjects were instructed in advance not to move or talk and to continue breathing normally during the CFT, which lasted for $2 \mathrm{~min}$.

\section{Measures}

Cortisol

Examinations started in the afternoon between 1:30 and 4:15 p.m. to control for circadian fluctuations (Smyth, Ockenfels, Gorin, Catley, Porter, et al., 1997). To further control for circaseptan alterations (Bodis, Boncz, \& Kriszbacher, in press; Lee, Lee, Lee, Cornélissen Otsuka, \& Halberg, 2003; Maschke, Harder, Cornélissen, Hecht, Otsuka, \& Halberg, 2003), both examinations were conducted with 2 weeks in between. Salivary samples were repeatedly collected with salivettes (Sarstedt, Sevelen, Switzerland) before, during, and after the interventions by collecting unstimulated whole saliva: immediately before the MIST-S and MIST-C instruction, during the third block, and up to $60 \mathrm{~min}$ after the completion of the MIST (Figure 1A). Subjects placed a salivette under the tongue and kept the head slightly inclined for $2 \mathrm{~min}$. Saliva samples were stored at $-20^{\circ} \mathrm{C}$ before the biochemical analysis took place. After thawing, saliva was centrifuged at 3000 rpm for 5 min before free cortisol was analyzed using an immunoassay with time-resolved fluorescence detection (Dressendorfer, Kirschbaum, Rohde, Stahl, \& Strasburger, 1992).

\section{Electrophysiological Measures}

The LifeShirt system 200 (Vivometrics, Ventura, CA) was used to measure cardiac and pulmonary activity. This ambulatory cardiopulmonary measurement device consists of a garment with two integrated inductive plethysmography (IP) bands surrounding the midthorax and midabdomen and a connected palm, which additionally saves electrocardiographic data measured by three electrodes. The device was recently evaluated and shown to possess a good accuracy of detection and timing of beat-to-beat values (Heilman \& Porges, 2007). After volume calibration of the IP bands by the subjects breathing repeatedly into a fixed volume bag $(800 \mathrm{cc})$, data recording started. The record was then examined for artifacts and edited manually to correct for ectopic beats and arrhythmias by using linear interpolation.

The corrected inter-beat-interval (IBI) allowed the calculation of HR. The association of IBI in relation to respiration allowed the measurement of respiratory sinus arrhythmia (RSA), indexing cardiovagal activity. HR and RSA were determined for 1-min intervals and averaged for 5-min intervals (e.g., for time $\times$ condition interaction effects). HR and RSA were determined using the VivoLogic 3.1.2 software package (Vivometrics). RSA was determined using the time-domain peak-valley method (Grossman, von Beek, \& Wientjes, 1990).

The effect of the CFT was measured as alteration in HR and RSA over $4 \mathrm{~min}$, from $1 \mathrm{~min}$ before onset until $1 \mathrm{~min}$ after completion of the CFT. The HR over the 1-min period preceding the CFT was set as baseline for two further measures with regard to the CFT. Maximum response of HR was determined as the relative difference between the baseline and peak response due to the CFT ( $\mathrm{CFT}_{\max } ;$ [\% change with respect to baseline HR]), and the latency of response was defined as the time interval from the first instance of three successively slowing beats below baseline until peak bradycardia (CFT latency; $_{\text {; }}$ sec]) (Khurana \& Wu, 2006). Additionally, since respiration alterations might affect RSA independent of cardiovagal involvement, tidal volume (Vt) and respiration rate $(\mathrm{fb})$ were extracted to be considered for statistical analysis.
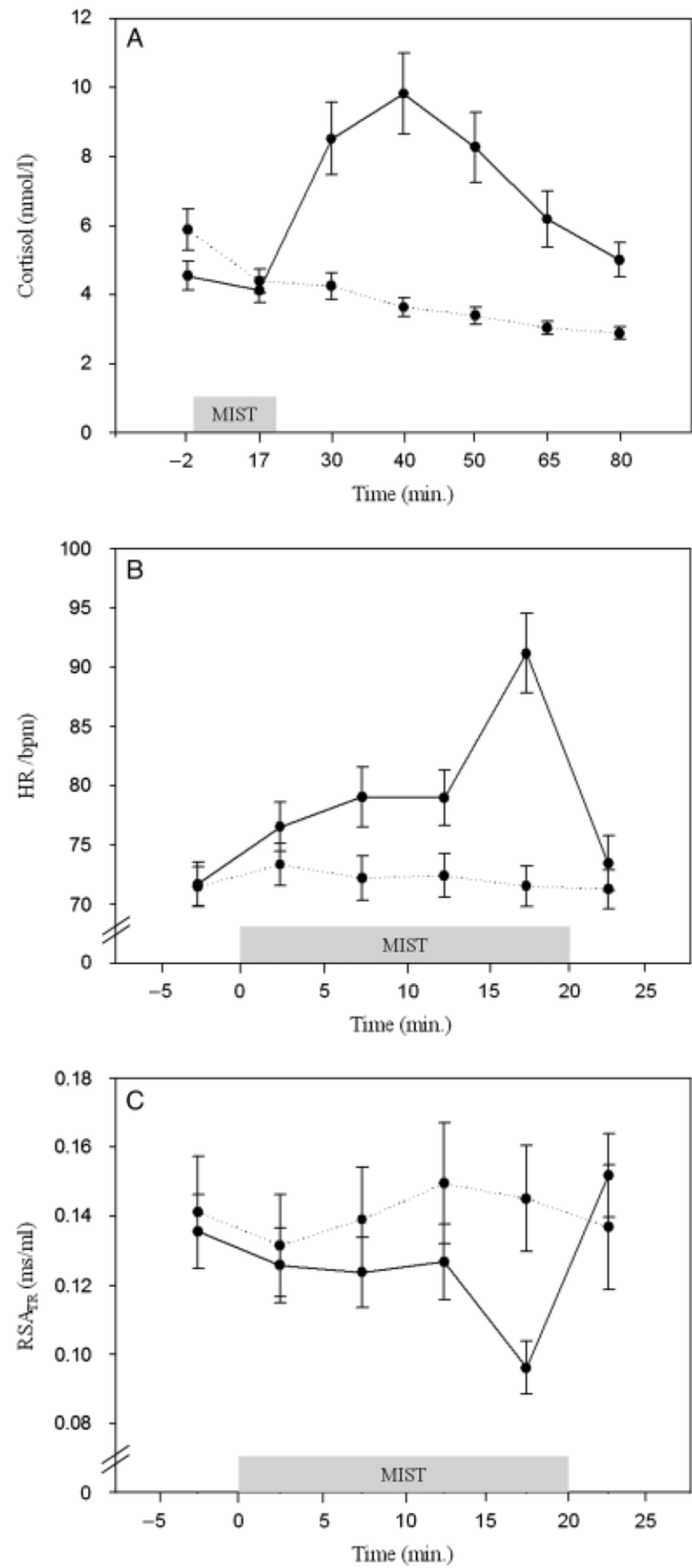

Figure 1. (A) Salivary cortisol concentration (nmol/l), (B) HR (beats per minute), and (C) RSA $\mathrm{TR}_{\mathrm{T}}(\mathrm{ms} / \mathrm{ml})$ during the stress (solid line) and control condition (dashed line). Values represent mean \pm standard error of the mean values.

\section{Psychological Measures}

Depressive mood was measured with a German version (Allgemeine Depressionsskala-Langform, ADS-L) (Hautzinger \& Bailer, 1992) of the Center for Epidemiologic Studies Depression Scale (CES-D) (Radloff, 1977) in order to exclude subjects with a possible depressive disorder (cut-off score $>23$ ). State mood was determined repeatedly, immediately before and after the MIST-S 
and MIST-C. To assess different aspects of mood, subjects filled out the Multidimensional Mood State Questionnaire (German original version: Multidimensionaler Befindlichkeitsfragebogen, MDBF) (Steyer, Schwenkmezger, Notz, \& Eid, 1997) consisting of three dimensions: 'good-bad mood,' 'calmness-nervousness,' and 'wakefulness-tiredness,' with higher values indicating good mood, calmness, and wakefulness. Furthermore, a visual analogue scale (VAS) was handed out before and after the stress and control condition asking subjects to rate how 'stressed' they felt at that moment. Further VAS asking for mood ratings (feeling stressed, exhausted, queasy, relaxed, good humored) and assessing pain were distributed $2 \mathrm{~min}$ before onset and after completion of the CFT to assess any changes in subjective sensation. The distributed questionnaires have been broadly used and have shown satisfactory internal consistency and validity.

\section{Data Analyses}

Analyses were performed using SPSS (17.0) software packages (SPSS, Chicago, IL). Homogeneity of variance was assessed using the Levene test. In addition to raw data, the trapezoid formula for total response (area under the curve with respect to the ground, $\mathrm{AUC}_{\mathrm{G}}$ ) and total change of response in consideration of individual baseline (area under the curve with respect to increase, $\mathrm{AUC}_{\mathrm{I}}$ ) were computed (La Marca, Nedeljkovic, Yuan, Maercker, \& Ehlert, 2010; Pruessner, Kirschbaum, Meinlschmid, \& Hellhammer, 2003; Scholz, La Marca, Nater, Aberle, Ehlert, et al., 2009). When referring to treatment order or condition (with regard to $\mathrm{AUC}_{\mathrm{G}}$ and $\mathrm{AUC}_{\mathrm{I}}$ ), paired Student's $t$-tests were used for comparisons between groups, while repeated-measures analyses of variance (ANOVA) were computed after Greenhouse-Geisser corrections to reveal possible interaction effects. As post hoc tests, we repeated the previously described calculations separately in each condition group. For assessment of associations between vagal function and biopsychological stress responses, we used a two-step procedure. We first calculated correlations to test for significant associations between vagal function (baseline, stress-induced inhibition, CFT-induced stimulation) and cortisol measures (baseline, stress-induced stimulation). Second, to avoid multiple testing, we only calculated linear regressions with significant vagal markers from step 1 as independent variables. Cortisol, cardiopulmonary, and psychological stress responses were used as dependent variables in separate regression models. Explained variance in regression models is reflected by $\mathrm{R}^{2}$. In terms of RSA, Vt and fb were controlled for in all statistical analyses due to significant alterations of Vt and fb during the MIST-S and the CFT. Therefore,

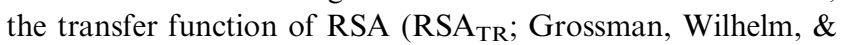
Spoerle, 2004; Grossman, Spoerle, \& Wilhelm, 2006; La Marca et al., 2010) was applied, while further controlling for the $\mathrm{AUC}_{\mathrm{I}}$ of fb during the MIST-S and/or CFT. In condition $\times$ time interactions, the effect size was determined by partial eta-square $\left(\right.$ partial eta $\left.{ }^{2}\right)$ reflecting small (.01), medium (.06), or large (.14) effect sizes (Green, Salkind, \& Akey, 2000). All analyses were two-tailed, with the level of significance set at $p<.05$.

\section{Results}

\section{Sample Characteristics}

Thirty-four healthy subjects agreed to participate in the study. One subject was excluded during the examination period because of acute illness on the day of the stress examination. Low amounts of saliva in some samples further reduced the available number of data points for cortisol (AUC $\left.\mathrm{I}_{\text {(MIST-S) }}: N=28\right)$. The mean age of participants was $M=24.06(S D=4.56$, range 19-34) and the mean BMI was $M=23.02$ ( $S D=2.93$, range 18.83-29.05). Depression scores were in a normal range of values $(M=7.84$, $S D=4.14$, range 2-21). Randomization resulted in two groups, with 16 subjects undergoing the control condition first and 17 subjects undergoing the stress condition first. The two groups did not differ with respect to demographic (BMI, age), physiological, and mood changes during the stress condition (all $p=$ n.s.). Referring to the control condition, the corresponding variables did not differ, with the exception of the wakefulness-tiredness dimension of the MDBF, whereas subjects participating in the control condition in the second session became less tired compared to subjects who started with the control condition $\left(\mathrm{t}_{31}=-2.93\right.$, $p=.006)$. The randomization was therefore successful.

\section{Responses to the Stress Task}

All 33 subjects completed all of the three blocks of the MIST-S and MIST-C.

\section{Cortisol Response}

Cortisol levels significantly increased during the stress condition as compared to the control condition (interaction condition by time: $F(2.27 / 56.69)=20.94 ; p<.001 ;$ partial eta $^{2}=.46 ;$ Figure $\left.1 \mathrm{~A}\right)$. Differences in AUC values further supported this finding (main effect of condition: Cortisol-AUC $\mathrm{G}_{\mathrm{G}}$ : $\mathrm{t}_{25}=-4.98, p<.001$; Cortisol-AUC $\left.\mathrm{I}_{\mathrm{I}}: \mathrm{t}_{25}=-5.58, p<.001\right)$. Post hoc testing separately in each condition revealed a significant increase over time during the MIST-S (main effect of time: $F(2.02 / 54.51)=16.59$; $p<.001$ ), and a significant decrease during the MIST-C, which is typical for the circadian course of cortisol (main effect of time: $F(1.83 / 45.65)=24.55 ; p<.001)$. At the peak response of cortisol 20 min after task completion (Figure 1A; min. 40), the MIST-S compared to the MIST-C resulted in a 2.64 times higher value.

\section{Electrophysiological Responses}

$H R$ significantly increased during the stress condition as compared to the control condition (interaction condition by time: $F(2.13 / 68.00)=39.34, p<.001$, partial eta ${ }^{2}=.55$; Figure 1B). Furthermore, this finding was supported by differences in AUC values (main effect of condition: HR-AUC ${ }_{\mathrm{G}}: \mathrm{t}_{32}=-3.53, p=.001$; HR$\left.\mathrm{AUC}_{\mathrm{I}}: \mathrm{t}_{32}=-5.33, p<.001\right)$. Notably, significant alterations were found in both conditions (main effect of time: MIST-S: $F(2.10 /$ $67.06)=42.10, p<.001$; MIST-C: $F(3.15 / 100.69)=4.46, p=.005)$.

In contrast to $\mathrm{HR}, R S A_{T R}$ levels significantly decreased during the MIST-S as compared to the MIST-C (interaction of condition by time: $\mathrm{F}(3.14 / 97.21)=10.41, p<.001$; partial eta $^{2}=.25$; Figure 1C). AUC measures, however, did not differ between conditions (main effect of condition: $\mathrm{RSA}_{\mathrm{TR}}-\mathrm{AUC}_{\mathrm{G}}: F(1.00 / 31.00)=1.53$, $\left.p=.23 ; \mathrm{RSA}_{\mathrm{TR}}-\mathrm{AUC}_{\mathrm{I}}: F(1.00 / 31.00)=2.08, p=.16\right)$. A significant decrease of $\mathrm{RSA}_{\mathrm{TR}}$ was found during the stress condition (main effect of time: $F(3.15 / 97.62)=10.71, p<.001$ ), while no significant alteration was found during the control condition (main effect of time: $F(3.23 / 100.17)=1.64, p=.18)$.

\section{Mood Responses}

ANOVAs revealed a significant difference in terms of mood (MDBF) between MIST-S and MIST-C (interaction condition by time: $F(1.00 / 32.00)=25.51, p<.001$, partial eta $^{2}=.44$; Table 1), with a significant worsening in mood during the stress (main effect of time: $F(1.00 / 32.00)=27.80, p<.001$ ) but not the control condition (main effect of time: $F(1.00 / 32.00)=.52$, $p=.48$ ), as revealed by post hoc testing. 
Table 1. Paired Student's t-test Between MIST-S and MIST-C Induced Changes in the Three Dimensions 'Mood,' 'Calmness,' and 'Wakefulness' of the MDBF, and the Visual Analog Scale 'Stressed'

\begin{tabular}{lcc}
\hline \hline & Diff $_{\text {MIST-C }}(S D)$ & Diff $_{\text {MIST-S }}(S D)$ \\
\hline Mood & $.21(1.69)$ & $-3.88^{* * * *}(4.22)$ \\
Calmness & $.42(2.17)$ & $-4.70^{* * * * *}(3.35)$ \\
Wakefulness & $.84(3.32)$ & $-.72^{*}(3.19)$ \\
Stressed & $-.59(1.89)$ & $2.93^{* * * * * 1}(3.15)$ \\
\hline \hline
\end{tabular}

Note: Values are means \pm standard deviations. Diff MIST-S $=$ values immediately after MIST-S minus baseline values; $\operatorname{Diff}_{\text {MIST-C }}=$ values immediately after MIST-C minus baseline values.

$* p<.05 ; * * * p<.001$.

Similarly, ANOVAs revealed a significant difference in calmness (MDBF) between the two conditions (interaction condition by time: $F(1.00 / 32.00)=52.30, p<.001$, partial eta $\left.{ }^{2}=.62\right)$, due to a significant decrease in calmness during the stress (main effect of time: $F(1.00 / 32.00)=64.90, p<.001)$ but not the control condition (main effect of time: $F(1.00 / 32.00)=1.27, p=.27$ ).

With regard to wakefulness (MDBF), the two conditions differed significantly (interaction condition by time: $F(1 / 31)=4.41, p=.044$, partial eta $\left.{ }^{2}=.12\right)$, although post hoc testing was unable to reveal either the MIST-S (main effect of time: $F(1.00 / 32.00)=1.71, p=.20$ ) or the MIST-C (main effect of time: $F(1.00 / 31.00)=2.06, p=.16$ ) to alter rated wakefulness.

Finally, the item feeling stressed (VAS) revealed a significant interaction effect (interaction condition by time: $F(1 / 32)=25.97$, $p<.001$, partial eta $\left.{ }^{2}=.45\right)$, with a significant increase during the MIST-S (main effect of time: $F(1 / 32)=28.53, p<.001$ ), but not during the MIST-C (main effect of time: $F(1 / 32)=3.25, p=.08$ ).

\section{Responses to the Cold Face Test}

The CFT induced a significant decrease in HR (main effect of time: $F(2.17 / 69.48)=39.94, p<.001$, partial eta $\left.{ }^{2}=.56\right)$ and, accordingly, a significant increase in $R S A_{T R}$ (main effect of time: $F(2.40 /$ $74.53)=10.73, p<.001$, partial eta ${ }^{2}=.26$ ), indicating a successful cardiovagal stimulation. The bradycardia during the CFT peaked after $\mathrm{M}\left(\mathrm{CFT}_{\text {latency }}\right)=29.85 \mathrm{sec}(S D=19.95 \mathrm{sec})$ and resulted in a maximum decrease of $\mathrm{M}\left(\mathrm{CFT}_{\max }\right)=24.80 \%(S D=7.64 \%)$. The cardiopulmonary changes during the CFT could not be attributed to subjective mood or pain changes, which did not alter significantly (main effect of time: $\mathrm{VAS}_{\text {stressed }} \mathrm{t}_{32}=.48, p=.63$; VAS $_{\text {exhausted }}: \mathrm{t}_{32}=.91, p=.37 ;$ VAS $_{\text {queasy }}: \mathrm{t}_{32}=1.47, p=.15$; VAS $_{\text {relaxed }}: \mathrm{t}_{32}=.73, p=.47 ; \mathrm{VAS}_{\text {good humoured }} \mathrm{t}_{32}=-.05$, $p=.96$; VAS $_{\text {pain }}: \mathrm{t}_{32}=-.09, p=.93$ ).

\section{Associations Between Vagal and HPA Function}

Vagal baseline. Bivariate correlations with baseline of HR and $\mathrm{RSA}_{\mathrm{TR}}$ revealed no significant relation to cortisol measured under baseline and stress conditions (Table 2).

Stress-induced vagal inhibition. Similar to vagal baseline, partial correlations controlling for fb changes during the MIST-S revealed no significant association between $\mathrm{AUC}_{\mathrm{I} \text { (MIST-S) }}$ of $\mathrm{RSA}_{\mathrm{TR}}$ and baseline or $\mathrm{AUC}_{\mathrm{I}(\mathrm{MIST}-\mathrm{S})}$ of cortisol (Table 2).

CFT-induced vagal stimulation. Partial correlations controlling for the $\mathrm{AUC}_{\mathrm{I}}$ of $\mathrm{fb}$ during the CFT showed no significant association between the $\mathrm{AUC}_{\mathrm{I}}$ of $\mathrm{RSA}_{\mathrm{TR}}$ during the CFTand the baseline
Table 2. Correlations of Measures of Vagal and Cortisol (Re-)Activity During Different Conditions, Controlling for $\mathrm{fb}$ Alterations, Where Appropriate

\begin{tabular}{|c|c|c|c|}
\hline \multirow[b]{2}{*}{ Vagal function } & \multirow[b]{2}{*}{ Vagal parameters } & \multicolumn{2}{|c|}{ Cortisol measures } \\
\hline & & Baseline & $\mathrm{AUC}_{\mathrm{I}(\mathrm{MIST}-\mathrm{S})}$ \\
\hline \multirow[t]{2}{*}{ Baseline } & $\mathrm{RSA}_{\mathrm{TR}}$ : baseline & .164 & .017 \\
\hline & HR: baseline & .094 & .034 \\
\hline Inhibition & $\mathrm{RSA}_{\mathrm{TR}}: \mathrm{AUC}_{\mathrm{I}(\mathrm{MIST}-\mathrm{S})}$ & -.050 & -.217 \\
\hline \multirow[t]{3}{*}{ Stimulation } & $\mathrm{RSA}_{\mathrm{TR}}: \mathrm{AUC}_{\mathrm{I}(\mathrm{CFT})}$ & -.171 & .015 \\
\hline & $\mathrm{CFT}_{\max }$ & .090 & -.032 \\
\hline & $\mathrm{CFT}_{\text {latency }}$ & -.024 & $.424^{*}$ \\
\hline
\end{tabular}

Note: Values are correlational coefficients $r$.

$* p<.05$.

or $\mathrm{AUC}_{\mathrm{I}}$ of cortisol during the MIST-S (Table 2). Similarly, bivariate correlations with $\mathrm{CFT}_{\max }$ showed no relation to cortisol. In contrast, bivariate correlations with $\mathrm{CFT}_{\text {latency }}$ revealed a significant positive association with $\mathrm{AUC}_{\mathrm{I}(\mathrm{MIST}-\mathrm{S})}$ of cortisol, but not with baseline. Subjects with longer latency until peak bradycardia showed a higher stress response of cortisol (Figure 2A). Based on the analysis of correlation, we conducted a linear regression analysis
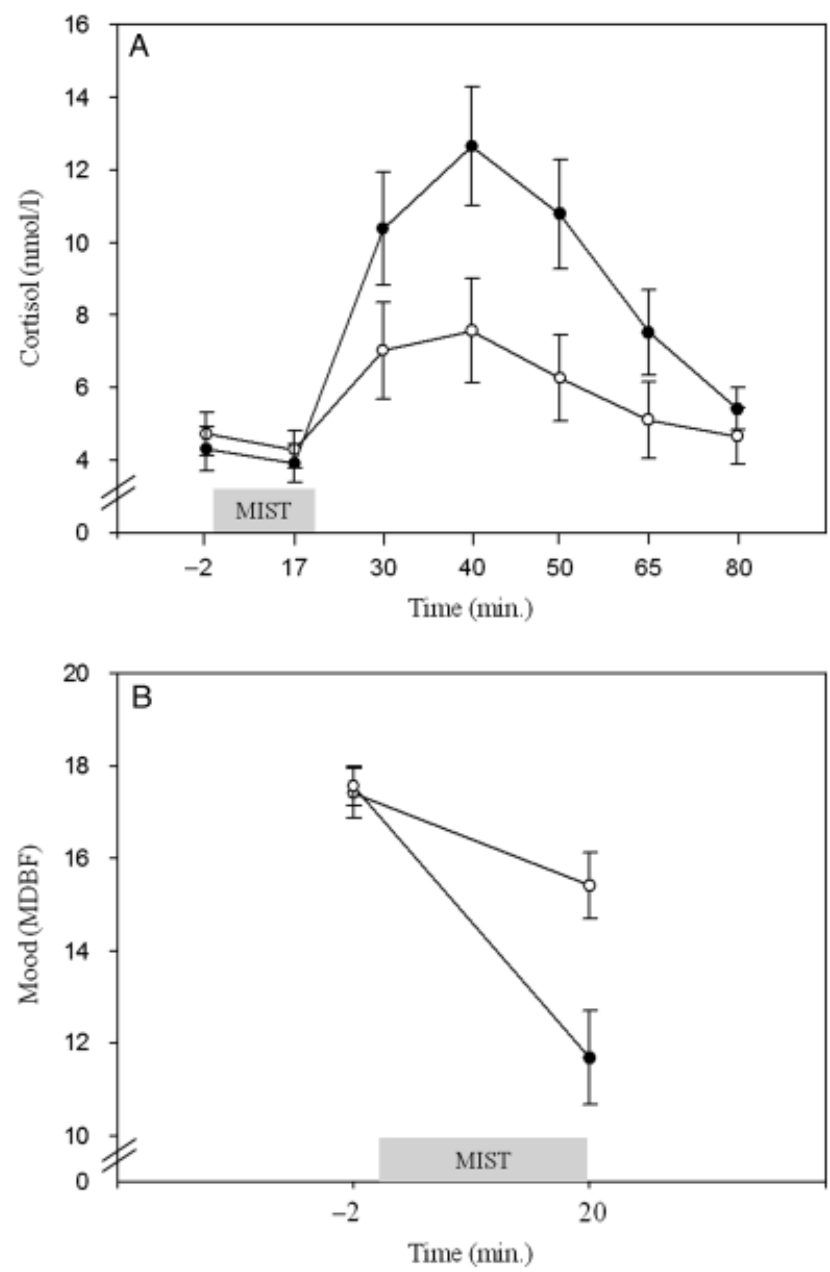

Figure 2. Stress reaction of (A) salivary cortisol and (B) mood in subjects with short (white circles) and long (black circles) latencies until peak bradycardia during the CFT ( $\mathrm{CFT}_{\text {latency }}$ ) determined after median split. Values represent mean \pm standard error of the mean values. 
Table 3. Hierarchical Regression Analyses for Associations Between CFT $T_{\text {latency }}$ and Stress Responses

\begin{tabular}{|c|c|c|c|c|}
\hline Variables entered & $\begin{array}{l}\text { Standardized } \\
\text { coefficient } \beta\end{array}$ & $t$ & $p$ & $\mathrm{R}^{2}$ change \\
\hline \multicolumn{5}{|l|}{$\mathrm{AUC}_{\mathrm{I} \text { (MIST-S) }}$ of cortisol } \\
\hline $\mathrm{CFT}_{\text {latency }}$ & .42 & 2.38 & .025 & .18 \\
\hline \multicolumn{5}{|l|}{$\mathrm{AUC}_{\mathrm{I}(\mathrm{MIST}-\mathrm{S})}$ of $\mathrm{HR}$} \\
\hline $\mathrm{CFT}_{\text {latency }}$ & .13 & .75 & .46 & .02 \\
\hline \multicolumn{5}{|l|}{$\mathrm{AUC}_{\mathrm{I}(\mathrm{MIST}-\mathrm{S})}$ of RSA $\mathrm{RR}_{\mathrm{TR}}$} \\
\hline $\mathrm{AUC}_{\mathrm{I}(\mathrm{MIST}-\mathrm{S}) \text { of } \mathrm{fb}}$ & -.54 & -3.49 & .002 & .29 \\
\hline $\mathrm{CFT}_{\text {latency }}$ & -.01 & -.08 & .94 & .00 \\
\hline \multicolumn{5}{|l|}{ Diff MIST-S in mood } \\
\hline $\mathrm{CFT}_{\text {latency }}$ & -.60 & -4.22 & $<.001$ & .36 \\
\hline \multicolumn{5}{|l|}{ Diff $_{\text {MIST-S in calmness }}$} \\
\hline $\mathrm{CFT}_{\text {latency }}$ & -.20 & -1.11 & .28 & .04 \\
\hline \multicolumn{5}{|l|}{ Diff $_{\text {MIST-S in wakefulness }}$} \\
\hline $\mathrm{CFT}_{\text {latency }}$ & -.20 & -1.12 & .27 & .04 \\
\hline \multicolumn{5}{|c|}{ Diff $_{\text {MIST-S }}$ in feeling stressed } \\
\hline $\mathrm{CFT}_{\text {latency }}$ & .12 & .65 & .52 & .01 \\
\hline
\end{tabular}

Note: We controlled for $\mathrm{fb}$ alterations where appropriate. Diff $\mathrm{MIST}_{\mathrm{S}}=$ values immediately after MIST-S minus baseline values; $\mathrm{AUC}_{\mathrm{I}(\mathrm{MIST}-\mathrm{S})}=$ area under the curve with respect to increase during the stress condition; $\mathrm{CFT}_{\text {latency }}=$ time from CFT onset until maximum bradycardia.

with $\mathrm{CFT}_{\text {latency }}$ as independent variable and $\mathrm{AUC}_{\mathrm{I}(\mathrm{MIST}-\mathrm{S})}$ of cortisol as dependent variable. $\mathrm{CFT}_{\text {latency }}$ explained $17.9 \%$ of total variance in $\mathrm{AUC}_{\mathrm{I}(\mathrm{MIST}-\mathrm{S})}$ of cortisol (Table 3).

\section{Associations Between CFT $T_{\text {latency }}$ and Cardiopulmonary and Psychological Stress Response}

After the first step identifying $\mathrm{CFT}_{\text {latency }}$ to be correlated with HPA function, in a second step, we were interested in identifying stress responses associated with $\mathrm{CFT}_{\text {latency }}$. Linear regression analyses were conducted entering $\mathrm{CFT}_{\text {latency }}$ as independent variable, and $\mathrm{AUC}_{\mathrm{I}}$ of $\mathrm{HR}, \mathrm{AUC}_{\mathrm{I}}$ of $\mathrm{RSA}_{\mathrm{TR}}$, or alterations in psychological measures in response to the MIST-S as dependent variables. $\mathrm{AUC}_{\mathrm{I}(\mathrm{MIST}-\mathrm{S})}$ of fb was controlled for when examining $\mathrm{AUC}_{\mathrm{I}(\mathrm{MIST}-\mathrm{S})}$ of $\mathrm{RSA}_{\mathrm{TR}}$. $\mathrm{CFT}_{\text {latency }}$ was not a significant predictor of the $\mathrm{AUC}_{\mathrm{I}}$ of $\mathrm{HR}$ and $\mathrm{RSA}_{\mathrm{TR}}$ in response to the MIST-S (Table 3). In contrast, $\mathrm{CFT}_{\text {latency }}$ as independent variables explained $36.5 \%$ of total variance in the stress response of mood. Therefore, longer latency until peak bradycardia was associated with worse mood after the MIST-S (Figure 2B).

\section{Discussion}

The main objective of the present study was to test the association between the function of the VN and the HPA axis, with a special focus on the reactivity of the two systems. In this context, longer latency to peak bradycardia during the CFT was associated with higher cortisol and more negative mood response to the stress task, while smaller maximal bradycardia was associated with more tiredness in response to the stress task. Furthermore, for the first time, the MIST-S was demonstrated to be an effective stress test for inducing a strong vagal inhibition, therefore increasing its validity to induce a multidimensional stress response.

Our findings replicate those of prior investigations showing an increase in cortisol concentration in response to the MIST-S, indicating an effective stress-induced stimulation of the HPA axis (Dedovic et al., 2005; Pruessner, Dedovic, Khalili-Mahani, Engert, Pruessner, et al., 2008; Soliman, O’Driscoll, Pruessner, Holahan, Boileau, Gagnon, \& Dagher, 2008). Furthermore, HR increased continuously throughout the stress condition, suggesting a successive increase in stress load from block to block, and recovered immediately after termination of the MIST-S. This is the first study to examine the effects of the MIST-S on the PSNS. RSA $_{\mathrm{TR}}$ decreased during the stress task, with a peak depression during the final block, indicating an effective stress-induced inhibition of the VN. Additionally, we found a significant increase in rated stress and a significant decrease in mood, wakefulness, and calmness ratings after the MIST-S, corresponding to prior studies (Pruessner, Champagne, Meaney, \& Dagher, 2004; Soliman et al., 2008).

The primary aim of the current study was to investigate the association between the function of the VN and the HPA axis. Therefore, in addition to baseline and stress-induced inhibition or stimulation parameters, the CFT was conducted to further collect data on induced stimulation of the VN. An inhibiting role of the VN on the HPA axis can be assumed (Thayer \& Sternberg, 2006). Nevertheless, evidence examining the relation of HPA axis and vagal function is contradictory, as measured by cortisol and HRV associations.

In the present study, we found a reduced cortisol response elicited by the MIST-S to be associated with a faster achievement of peak bradycardia (i.e., short $\mathrm{CFT}_{\text {latency }}$ ), indicating an inverse relationship between the function of the VN and the HPA axis. This inverse association is supported by Johnsen et al. (2002), who reported that a group of healthy men with higher baseline HRV (median split) showed lower levels of cortisol in response to stressful cognitive tasks compared to a group with lower baseline HRV. Similarly, Doussard-Roosevelt et al. (2003) found an inverse relationship between stress responses of RSA and cortisol to negative affect tasks in kindergarten children. Thus, a higher decrease in RSA was associated with a higher increase in cortisol in response to the emotional tasks. Further support for an inverse relation between the two systems is reported by Thayer et al. (2006). In a large sample of healthy men, they found a weak inverse relationship between HRV assessed over repeated hours $(<24 \mathrm{~h})$ and overnight urinary cortisol after a working day, variables that therefore possibly represent everyday-life-stress measurement (e.g., work-related stress). Hence, subjects with higher HRV revealed lower urinary cortisol levels.

In the present study, we found no significant correlation between the baseline or the $\mathrm{AUC}_{\mathrm{I}(\mathrm{MIST}-\mathrm{S})}$ of $\mathrm{RSA}_{\mathrm{TR}}$ and the AUC $_{\text {I(MIST-S) }}$ of cortisol. This is in line with most reports (Altemus et al., 2001; Cacioppo et al., 1995; Doussard-Roosevelt et al., 2003; Gunnar et al., 1995; Heilman et al., 2008), which also found no relation between HRV and cortisol in response to different stressors (social, psychological, physical stress tasks).

One study (Gunnar et al., 1995), even found a significant positive association between baseline RSA and stress response of cortisol, with newborns with higher baseline levels of RSA showing higher cortisol levels in response to the stress task. The authors argued, referring to Porges (1991), that higher vagal tone should show greater physiological responses to stressors. In addition, we found no association between baseline levels of RSA $_{T R}$ and cortisol, which is also in line with other studies (Gunnar et al., 1995; Johnsen et al., 2002).

Taken together, the rarely reported association between HRV and cortisol is mostly limited to stress-induced alterations, especially of the HPA axis. While we were unable to support this association, we found a strong association between $\mathrm{CFT}_{\text {latency }}$ as an indicator of the CFT-induced stimulation of the VN and cortisol stress response. There are different possible explanations 
for this inconsistency. First, several studies examining associations between both HPA axis and vagal function under stress reveal no significant alterations of either cortisol or HRV in response to the applied stress task (e.g., Altemus et al., 2001; Heilman et al., 2008). Second, HRV possesses huge interindividual variations (e.g., Grossman \& Taylor, 2007), and is therefore less adequately applicable for interindividual comparisons, but is better for intraindividual comparisons. Therefore, the CFT-induced vagal stimulation response might be more adequate for interindividual comparisons. Third, due to its high adaptation speed to changing demands, vagal contribution in general is of highest importance at the beginning of a demand, since it is the first and fastest way to adapt to a stimulus (Porges, 2001). This is especially true for the CFT, which imitates the diving reflex by directly provoking a lowering in heart activity through trigeminal-vagal stimulation. Therefore, the latency until peak bradycardia during the CFT might be a better and more sensitive index of vagal participation or influence than the more raw estimation of vagal function estimated by baseline or changes of averaged levels of $\mathrm{RSA}_{\mathrm{TR}}$.

Further indirect support for an inverse relationship between the function of the VN and the HPA axis, but without examining cortisol and cardiopulmonary data concomitantly, is provided by O'Keane, Dinan, Scott, and Corcoran (2005), who examined responses to the corticotropin-releasing-hormone $(\mathrm{CRH})$ challenge test in patients with chronic depression before and after three months of treatment with vagus nerve stimulation (VNS), an invasive treatment shown to have good effects in treatmentresistant depression (Rush et al., 2000). They reported significantly increased responses of adrenocorticotropic hormone (ACTH) and cortisol before treatment compared to intraindividual posttreatment responses, and in comparison to a control group. The negative association might point to bidirectional connections between vagal nuclei in the medulla oblongata and the hypothalamus (Benarroch, 1997; Palkovits, 1999). Furthermore, evidence from imaging studies supports interconnections between CAN structures, the hypothalamus, and the vagus nerve. For example, the salivary cortisol in response to stress has been shown to be positively associated with hypothalamic activity (Åhs, Furmark, Michelgård, Långström, Appel, et al., 2006), and negatively correlated with medial PFC (Åhs et al., 2006; Kern, Oakes, Stone, McAuliff, Kirschbaum, \& Davidson, 2008), suggesting a possible phasic loss of inhibitory control of the medial PFC over subcortical regions during stress. Similarly, under stressful conditions, associations between High Frequency (HF) HRV (Task Force, 1996) as an index of cardiovagal activity and the activity of several structures of the CAN were demonstrated (Gianaros, Van Der Veen, \& Jennings, 2004; Lane, McRae, Reiman, Chen, Ahern, \& Thayer, 2009; Matthews, Paulus, Simmons, Nelesen, \& Dimsdale, 2004).

Analyses of the role of vagal function during acute stress revealed a protective role not only with regard to cortisol but also to mood. Subjects with a longer latency to peak bradycardia during the CFT showed a more negative affect in response to the stressor. This is in line with Ingjaldsson, Laberg, and Thayer (2003), who found a negative correlation of HRV with negative mood but a positive correlation of HRV with positive mood in a population of alcoholics and healthy controls (Ingjaldsson et al., 2003). Furthermore, we found that subjects with smaller relative bradycardia (i.e., lower $\mathrm{CFT}_{\max }$ values) were more tired after the stress task compared to subjects with stronger bradycardia (i.e., higher $\mathrm{CFT}_{\max }$ values). This is in line with the reported positive association between HRV and efficient attentional regulation (Johnsen, Thayer, Laberg, Wormnes, Raadal, et al., 2003), which might offer an explanation for the present findings. Additional support for this interpretation is provided by the findings of an inverse course of effort and HRV in burnout patients and healthy controls during a repeated Stroop color word task over one day (Zanstra, Schellenkens, Schaap, \& Kooistra, 2006). Controls showed a decrease in HRV and effort over the day, while at the same time, burnout patients revealed an increase in HRV, effort, and tiredness.

We found no significant association between the CFT parameters and the cardiopulmonary stress response. One possible explanation could relate to the high interindividual differences in $\mathrm{RSA}_{\mathrm{TR}}$ mentioned previously (Grossman \& Taylor, 2007). Furthermore, the CFT parameters indicate a short-term dynamic vagal characteristic. This characteristic might have more influence on the HPA axis response, while vagal baseline, as a less dynamic characteristic of the vagus nerve, was more predictive of the $\mathrm{AUC}_{\mathrm{I}(\mathrm{MIST}-\mathrm{S})}$ of $\mathrm{RSA}_{\mathrm{TR}}$ (data not shown). Referring to $\mathrm{HR}$, the $\mathrm{AUC}_{\mathrm{I}(\mathrm{MIST}-\mathrm{S})}$ of $\mathrm{RSA}_{\mathrm{TR}}$ was negatively associated with the $\mathrm{AUC}_{\mathrm{I} \text { (MIST-S) }}$ of HR (data not shown). Therefore, this finding indicates that the HR response might be more dependent on an acute, concomitant vagal response.

With regard to the CFT, it is proposed as a method to test vagal function (Khurana \& Wu, 2006). Although, unfortunately, no study has examined the neural associations of latency and maximum bradycardia, the CFT was found to elicit several activity alterations in different CAN structures (Brown, Sanya, \& Hilz, 2003; Harper, Macey, Henderson, Woo, Macey, et al., 2003). Interestingly, low vagal response during the CFT was associated with depressed mood (Hughes \& Stoney, 2000), indicating an impaired vagal regulation in depression, which is often reported to show increased cortisol concentrations (Wong, Kling, Munson, Listwak, Licinio, et al., 2000) and decreased vagal tone (van der Kooy, van Hout, van Marwijk, de Haan, Stehouwer, \& Beekman, 2006). Similar results were found for hostility (Ruiz, Uchino, \& Smith, 2006), while an inverse association between anger control and cortisol reactivity was also determined (Gouin, Kiecolt-Glaser, Malarkay, \& Glaser, 2008). Furthermore, a missed vagal responsiveness to mental challenge was found in posttraumatic stress disorder (Sahar, Shalev, \& Porges, 2001), a disorder which shows an attenuated cortisol response to acute stress (MacMillan, Georgiades, Duku, Steiner, Niec, et al., 2009).

Since a faster response during the CFT is associated with a reduced cortisol response to stress, paralleled by better mood, it seems appealing to interpret a fast vagal response as healthprotective. Explanations for a beneficial interpretation of the results is provided by the function of the diving reflex per se, which elicits bradycardia as a protective strategy to conserve oxygen (Rozloznik, Paton, \& Dutschmann, 2009), the cardioprotective role of vagal activity (Fallen, 2005), the association between lower vagal activity and morbidity (Thayer \& Brosschot, 2005; Thayer \& Lane, 2007), and the therapeutic effects of VNS in therapy-resistant patients (Milby, Halpern, \& Baltuch, 2008). Furthermore, the protective interpretation of vagal functionality during stress is in line with the assumption of vagal activity as being a type of resource when demands of the environment request emotional regulation (Thayer \& Lane, 2009). Evidence for the interpretation of the $\mathrm{VN}$ as a resource was provided among others by Ruiz-Padial, Sollers, Vila, and Thayer (2003), who demonstrated a more differentiated emotional startle 
response to emotional pictures in subjects with high HRV compared to the group with low HRV. Furthermore, HRV was found to be positively associated with positive mood and negatively associated with negative mood (Ingjaldsson et al., 2003).

Although our hypotheses were mostly supported, the current study holds a number of limitations. First, we examined a small sample size, including only healthy and medication-free males. Therefore, results are restricted to a group of healthy, well-educated, young men and cannot be generalized to women or the general population. Moreover, the CFT in this study was used to determine vagal function (i.e., acute vagal stimulation), suggested by us to be indicative of the flexibility of the whole CAN. It should be mentioned on a critical note that CFT responses are initially mediated by the trigeminal nerve, and, therefore, responses could also be influenced by the latter responsiveness. Despite these limitations, however, our study does have a number of methodological strong points. First, we examined several vagal characteristics by examining not only vagal baseline, but also reactivity to the CFT (acute vagal stimulation) and the MIST-S (acute vagal inhibition). Second, by favoring the MIST$\mathrm{S}$ over other strong stressors known to induce a multidimensional stress response (e.g., Trier Social Stress Test, TSST; Kirschbaum, Pirke, \& Hellhammer, 1993), several potentially disruptive factors such as postural changes or walking (Chan, Lin, Chao, \& Lin, 2007; Nater, La Marca, Florin, Moses, Langhans, Koller, \& Ehlert, 2006), and vocalization (Bernardi, Wdowczyk-Szulc, Valenti, Castoldi, Passino, et al., 2000; Sloan, Korten, \& Myers, 1991) were controlled for. Therefore, the MIST-S seems particularly useful to investigate the relationship between different response variables that are not equally susceptible to the potentially disruptive factors. Notably, additional controlling for credibility of the stress task ("How much do you believe that your bad performance was due to a bug in the program?"; 10-point Likert scale, distributed at the end of the stress examination) did not significantly change any of the reported associations.

In summary, our results underline the validity of the MIST-S to provoke a multidimensional stress response including vagal inhibition. The inverse relationship between the vagal function measured during the CFT and stress responses of cortisol and mood support the assumption that the HPA axis plays an important role linking lower vagal function and morbidity. Furthermore, the CFT might be applied as an index to examine biopsychological stress reactivity of individuals. Moreover, we speculate that vagal baseline might reflect the tonic state of the interconnected CAN, while the CFT response might reflect the dynamic capacity of the organism to respond to rapidly changing environmental demands. Therefore, the CFT might be an interesting addition in the measurement of vagal function. However, the mechanisms underlying latency of bradycardia during the CFT are not entirely understood, and, therefore, further research is needed. The association between CFT-induced vagal stimulation and other HPA axis alterations, e.g., in response to other stressors or during awakening or the whole day, should be examined in future studies. Moreover, the reported association should be examined in subjects with (sub-)clinical somatic and mental disorders in prospective studies in order to gain further insight into the role of the HPA axis linking lower vagal function with morbidity.

\section{REFERENCES}

Åhs, F., Furmark, T., Michelgård, Å., Långström, B., Appel, L., Wolf, O. T., et al. (2006). Hypothalamic blood flow correlates positively with stress-induced cortisol levels in subjects with social anxiety disorder. Psychosomatic Medicine, 68, 859-862.

Altemus, M., Redwine, L. S., Leong, Y. M., Frye, C. A., Porges, S. W., \& Carter, C. S. (2001). Response to laboratory psychosocial stress in postpartum women. Psychosomatic Medicine, 63, 814-821.

Arnold, R. W. (1999). The human heart rate response profiles to five vagal maneuvers. Yale Journal of Biology and Medicine, 72, 237-244.

Benarroch, E. E. (1997). Central autonomic network: Functional organization and clinical correlations. Armonk, NY: Futura Publishing Company, Inc.

Bernardi, L., Wdowczyk-Szulc, J., Valenti, C., Castoldi, S., Passino, C., Spadacini, G., \& Sleight, P. (2000). Effects of controlled breathing, mental activity and mental stress with or without verbalization on heart rate variability. Journal of the American College of Cardiology, $35,1462-1469$.

Bernstein, A. L., Barkan, H., \& Hess, T. (2006). Vagus nerve stimulation therapy for pharmacoresistant epilepsy: Effect on health care utilization. Epilepsy \& Behavior, 10, 134-137.

Bodis, J., Boncz, I., \& Kriszbacher, I. (in press). Permanent stress may be the trigger of an acute myocardial infarction on the first work-day of the week. International Journal of Cardiology.

Brown, C. M., Sanya, E. O., \& Hilz, M. J. (2003). Effect of cold face stimulation on cerebral blood flow in humans. Brain Research Bulletin, 61, 81-86.

Cacioppo, J. T., Malarkey, W. B., Kiegolt-Glaser, J. K., Uchino, B. N., Sgoutas-Emch, S. A., Sheridan, J. F., et al. (1995). Heterogeneity in neuroendocrine and immune responses to brief psychological stressors as a function of autonomic cardiac activation. Psychosomatic Medicine, 57, 154-164.

Chan, H. L., Lin, M. A., Chao, P. K., \& Lin, C. H. (2007). Correlates of the shift in heart rate variability with postures and walking by timefrequency analysis. Computer Methods and Programs in Biomedicine, $86,124-130$.
Christensen, M. V., \& Kessing, L. V. (2001). The hypothalamo-pituitaryadrenal axis in major affective disorder: A review. Nordic Journal of Psychiatry, 55, 359-363.

Dedovic, K., Renwick, R., Khalili-Mahani, N., Engert, V., Lupien, S. J., \& Pruessner, J. C. (2005). The Montreal Imaging Stress Task: Using functional imaging to investigate the effects of perceiving and processing psychosocial stress in the human brain. Journal of Psychiatry \& Neuroscience, 30, 319-325.

Doussard-Roosevelt, J. A., Montgomery, L. A., \& Proges, S. W. (2003). Short-term stability of physiological measures in kindergarten children: Respiratory sinus arrhythmia, heart period, and cortisol. Developmental Psychobiology, 43, 230-242.

Dressendorfer, R. A., Kirschbaum, C., Rohde, W., Stahl, F., \& Strasburger, C. J. (1992). Synthesis of a cortisol-biotin conjugate and evaluation as a tracer in an immunoassay for salivary cortisol measurement. The Journal of Steroid Biochemistry and Molecular Biology, 43, 683-692.

Fallen, E. L. (2005). Vagal afferent stimulation as a cardioprotective strategy? Introducing the concept. Annals of Noninvasive Electrocardiology, 10, 441-446.

Gianaros, P. J., Van Der Veen, F. M., \& Jennings, J. R. (2004). Regional cerebral blood flow correlates with heart period and high-frequency heart period variability during working-memory tasks: Implications for the cortical and subcortical regulation or cardiac autonomic activity. Psychophysiology, 41, 521-530.

Girdler, S. S., Maixner, W., Naftel, H. A., Stewart, P. W., Moretz, R. L., \& Light, K. C. (2005). Cigarette smoking, stress-induced analgesia and pain perception in men and women. Pain, 114, 372-385.

Gouin, J. F., Kiecolt-Glaser, J. K., Malarkay, W. B., \& Glaser, R. (2008). The influence of anger expression on wound healing. Brain, Behavior, and Immunity, 22, 699-708.

Green, S. B., Salkind, N. J., \& Akey, T. M. (2000). Using SPSS for Windows (2nd ed). Englewood Cliffs, NJ: Prentice Hall.

Grossman, P., von Beek, J., \& Wientjes, C. (1990). A comparison of three quantification methods for estimation of respiratory sinus arrhythmia. Psychophysiology, 27, 702-714. 
Grossman, P., Wilhelm, F. H., \& Spoerle, M. (2004). Respiratory sinus arrhythmia, cardiac vagal control, and daily activity. American Journal of Physiology, Heart \& Circulatory Physiology, 287, H728-H734.

Grossman, P., Spoerle, M., \& Wilhelm, F. H. (2006). Reliability of respiratory tidal volume estimation by means of ambulatory inductive plethysmography. Biomedical Sciences Instrumentation, 42, 193-198.

Grossman, P., \& Taylor, E. W. (2007). Toward understanding respiratory sinus arrhythmia: Relations to cardiac vagal tone, evolution and biobehavioral functions. Biological Psychology, 74, 263-285.

Gunnar, M. R., Porter, F. L., Wolf, C. M., Rigatsu, J., \& Larson, M. C. (1995). Neonatal stress reactivity: Prediction to later emotional temperament. Child Development, 66, 1-13.

Harper, R. M., Macey, P. M., Henderson, L. A., Woo, M. A., Macey, K. E., Frysinger, R. C., et al. (2003). fMRI responses to cold pressor challenges in control and obstructive sleep apnea subjects. Journal of Applied Physiology, 94, 1583-1595.

Hautzinger, M., \& Bailer, M. (1992). Allgemeine Depressions Skala. Manual. Konstanz und Mainz: Beltz.

Heilman, K. J., Bal, E., Bazhenova, O. V., Sorokin, Y., Perlman, S. B., Hanley, M. C., \& Porges, S. W. (2008). Physiological responses to social and physical challenges in children: Quantifying mechanisms supporting social engagement and mobilization behaviors. Developmental Psychobiology, 50, 171-182.

Heilman, K. J., \& Porges, S. W. (2007). Accuracy of the LifeShirt ${ }^{\circledR}$ (Vivometrics) in the detection of cardiac rhythms. Biological Psychology, 75, 300-305.

Hord, E. D., Evans, M. S., Mueed, S., Adamolekun, B., \& Naritoku, D. K. (2003). The effect of vagus nerve stimulation on migraines. The Journal of Pain, 4, 530-534.

Hughes, J. W., \& Stoney, C. M. (2000). Depressed mood is related to high-frequency heart rate variability during stressors. Psychosomatic Medicine, 62, 796-803.

Ingjaldsson, J. T., Laberg, J. C., \& Thayer, J. F. (2003). Reduced heart rate variability in chronic alcohol abuse: Relationship with negative mood, chronic thought suppression, and compulsive drinking. Biological Psychiatry, 54, 1427-1436.

Johnsen, B. H., Hansen, A. L., Sollers III, J. J., Murison, R., \& Thayer, J. F. (2002). Heart rate variability is inversely related to cortisol reactivity during cognitive stress. Psychosomatic Medicine, 64, 148.

Johnsen, B. H., Thayer, J. F., Laberg, J. C., Wormnes, B., Raadal, M., Skaret, E., et al. (2003). Attentional and physiological characteristics of patients with dental anxiety. Journal of Anxiety Disorders, 17, 75-87.

Kern, S., Oakes, T. R., Stone, Ch. K., McAuliff, E. M., Kirschbaum, C., \& Davidson, R. J. (2008). Glucose metabolic changes in the prefrontal cortex are associated with HPA axis response to a psychosocial stressor. Psychoneuroendocrinology, 33, 517-529.

Khurana, R. K. (2007). Cold face test: Adrenergic phase. Clinical Autonomic Research, 17, 211-216.

Khurana, R. K., Watabiki, S., Hebel, J. R., Toro, R., \& Nelson, E. (1980). Cold face test in the assessment of trigeminal-brainstem-vagal function in humans. Annals of Neurology, 7, 144-149.

Khurana, R. K., \& Wu, R. (2006). The cold face test: A non-baroreflex mediated test of cardiac vagal function. Clinical Autonomic Research, 16, 202-207.

Kirschbaum, C., Kudielka, B. M., Gaab, J., Schommer, N. C., \& Hellhammer, D. H. (1999). Impact of gender, menstrual cycle phase, and oral contraceptives on the activity of the hypothalamus-pituitary-adrenal axis. Psychosomatic Medicine, 61, 154-162.

Kirschbaum, C., Pirke, K. M., \& Hellhammer, D. H. (1993). The 'Trier Social Stress Test' - A tool for investigating psychobiological stress responses in a laboratory setting. Neuropsychobiology, 28, 76-81.

La Marca, R., Nedeljkovic, M., Yuan, L., Maercker, A., \& Ehlert, U. (2010). Effects of auricular electrical stimulation on vagal activity in healthy men: Evidence from a three-armed randomized trial. Clinical Science, $118,537-546$

Lane, R. D., McRae, K., Reiman, E. M., Chen, K., Ahern, G. L., \& Thayer, J. F. (2009). Neural correlates of heart rate variability during emotion. NeuroImage, 44, 213-222.

Lee, M. S., Lee, J. S., Lee, J. Y., Cornélissen, G., Otsuka, K., \& Halberg, F. (2003). About 7-day (circaseptan) and circadian changes in cold pressor test (CPT). Biomedicine \& Pharmacotherapy, 57, 39s-44s.

MacMillan, H. L., Georgiades, K., Duku, E. K., Steiner, M., Niec, A., Tanaka, M., et al. (2009). Cortisol response to stress in female youths exposed to childhood maltreatment: Results of the youth mood project. Biological Psychiatry, 66, 62-68.
Maschke, C., Harder, J., Cornélissen, G., Hecht, K., Otsuka, K., \& Halberg, F. (2003). Chronoecoepidemiology of "strain": Infradian chronomics of urinary cortisol and catecholamines during nightly exposure to noise. Biomedicine \& Pharmacotherapy, 57, 126s-135s.

Matthews, S. C., Paulus, M. P., Simmons, A. N., Nelesen, R. A., \& Dimsdale, J. E. (2004). Functional subdivisions within anterior cingulate cortex and their relationship to autonomic nervous system function. NeuroImage, 22, 1151-1156.

Merrill, C. A., Jonsson, M. A., Minthon, L., Ejnell, H., C-son Silander, H., Blennow, K., et al. (2006). Vagus nerve stimulation in patients with Alzheimer's disease: Additional follow-up results of a pilot study through 1 year. The Journal of Clinical Psychiatry, 67, 1171-1178.

Milby, A. H., Halpern, C. H., \& Baltuch, G. H. (2008). Vagus nerve stimulation for epilepsy and depression. Neurotherapeutics, 5, 75-85.

Nater, U. M., La Marca, R., Florin, L., Moses, A., Langhans, W., Koller, M. M., \& Ehlert, U. (2006). Stress-induced changes in human salivary alpha-amylase activity - Associations with adrenergic activity. Psychoneuroendocrinology, 31, 49-58.

O'Keane, V., Dinan, T. G., Scott, L., \& Corcoran, C. (2005). Changes in hypothalamic-pituitary-adrenal axis measures after vagus nerve stimulation therapy in chronic depression. Biological Psychiatry, 58, 963-968.

Palkovits, M. (1999). Interconnections between the neuroendocrine hypothalamus and the central autonomic system. Frontiers in Neuroendocrinology, 20, 270-295.

Porges, S. W. (1991). Vagal tone: An autonomic mediator of affect. In J. Garber \& K. A. Dodge (Eds.), The development of emotion regulation and dysregulation (pp. 111-128). New York: Cambridge University Press.

Porges, S. W. (2001). The polyvagal theory: Phylogenetic substrates of a social nervous system. International Journal of Psychophysiology, 42, 123-146.

Pruessner, J. C., Champagne, F., Meaney, M. J., \& Dagher, A. (2004). Dopamine release in response to a psychological stress in humans and its relationship to early life maternal care: A positron emission tomography study using $\left[{ }^{11} \mathrm{C}\right]$ Raclopride. The Journal of Neuroscience, $24,2825-2831$

Pruessner, J. C., Dedovic, K., Khalili-Mahani, N., Engert, V., Pruessner, M., Buss, C., et al. (2008). Deactivation of the limbic system during acute psychosocial stress: Evidence from positron emission tomography and functional magnetic resonance imaging studies. Biological Psychiatry, 63, 234-240.

Pruessner, J. C., Kirschbaum, C., Meinlschmid, G., \& Hellhammer, D. H. (2003). Two formulas for computation of the area under the curve represent measures of total hormone concentration versus time-dependent change. Psychoneuroendocrinology, 28, 916-931.

Radloff, L. S. (1977). The CES-D scale: A self-report depression scale for research in the general population. Applied Psychological Measurement, 3, 385-401.

Rozloznik, M., Paton, J. F., \& Dutschmann, R. (2009). Repetitive paired stimulation of nasotrigeminal and peripheral chemoreceptor afferents cause progressive potentiation of the diving bradycardia. American Journal of Physiology-Regulatory, Integrative and Comparative Physiology, 296, R80-R87.

Ruiz, J. M., Uchino, B. N., \& Smith, T. W. (2006). Hostility and sex differences in the magnitude, duration, and determinants of heart rate response to forehead cold pressor: Parasympathetic aspects of risk. International Journal of Psychophysiology, 60, 274-283.

Ruiz-Padial, E., Sollers III, J. J., Vila, J., \& Thayer, J. F. (2003). The rhythm of the heart in the blink of an eye: Emotion-modulated startle magnitude covaries with heart rate variability. Psychophysiology, 40, $306-313$.

Rush, A. J., George, M. S., Sackeim, H. A., Marangell, L. B., Husain, M. M., Giller, C., et al. (2000). Vagus nerve stimulation (VNS) for treatment-resistant depression: A multicenter study. Biological Psychiatry, 47, 276-286.

Sahar, T., Shalev, A. Y., \& Porges, S. W. (2001). Vagal modulation of responses to mental challenge in posttraumatic stress disorder. Biological Psychiatry, 49, 637-643.

Sato, N., Myake, S., Akatsu, J., \& Kumashiro, M. (1995). Power spectral analysis of heart rate variability in healthy young women during the normal menstrual cycle. Psychosomatic Medicine, 57, 331-335.

Scholz, U., La Marca, R., Nater, U. M., Aberle, I., Ehlert, U., Hornung, R., et al. (2009). Go no-go performance under psychosocial stress: Beneficial effects of implementation intentions. Neurobiology of Learning and Memory, 91, 89-92. 
Sloan, R. P., Korten, J. B., \& Myers, M. M. (1991). Components of heart rate reactivity during mental arithmetic with and without speaking. Physiology and Behaviour, 50, 1039-1045.

Smyth, J. M., Ockenfels, M. C., Gorin, A. A., Catley, D., Porter, L. S., Kirschbaum, C., et al. (1997). Individual differences in the diurnal cycle of cortisol. Psychoneuroendocrinology, 22, 89-105.

Soliman, A., O'Driscoll, G. A., Pruessner, J., Holahan, A. V., Boileau, I., Gagnon, D., \& Dagher, A. (2008). Stress-induced dopamine release in humans at risk of psychosis: A $\left[{ }^{11} \mathrm{C}\right]$ Raclopride PET study. $\mathrm{Ne}$ uropsychopharmacology, 33, 2033-2041.

Steyer, R., Schwenkmezger, P., Notz, P., \& Eid, M. (1997). Der Mehrdimensionale Befindlichkeitsfragebogen (MDBF). Handanweisung. Göttingen: Hogrefe.

Task Force of the European Society of Cardiology and the North American Society of Pacing and Electrophysiology (1996). Heart rate variability-Standards and measurement, physiological interpretation and clinical use. Circulation, 93, 1043-1065.

Thayer, J. F. (2007). What the heart says to the brain (and vice versa) and why we should listen. Psychological Topics, 16, 241-250.

Thayer, J. F., \& Brosschot, J. F. (2005). Psychosomatics and psychopathology: Looking up and down from the brain. Psychoneuroendocrinology, 30, 1050-1058.

Thayer, J. F., \& Friedman, B. H. (2002). Stop that! Inhibition, sensitization, and their neurovisceral concomitants. Scandinavian Journal of Psychology, 43, 123-130.

Thayer, J. F., Hall, M., Sollers III, J. J., \& Fischer, J. E. (2006). Alcohol use, urinary cortisol, and heart rate variability in apparently healthy men: Evidence for impaired inhibitory control of the HPA axis in heavy drinkers. International Journal of Psychophysiology, 56, 244-250.

Thayer, J. F., \& Lane, R. D. (2000). A model of neurovisceral integration in emotion regulation and dysregulation. Journal of Affective Disorders, 61, 201-216.
Thayer, J. F., \& Lane, R. D. (2007). The role of vagal function in the risk for cardiovascular disease and mortality. Biological Psychology, 74, 224-242.

Thayer, J. F., \& Lane, R. D. (2009). Claude Bernard and the heart-brain connection: Further elaboration of a model of neurovisceral integration. Neuroscience and Biobehavioral Reviews, 33, 81-88.

Thayer, J. F., \& Sternberg, E. (2006). Beyond heart rate variability: Vagal regulation of allostatic systems. Annals of the New York Academy of Sciences, 1088, 361-372.

Tsigos, C., \& Chrousos, G. P. (1994). Physiology of the hypothalamuspituitary-adrenal axis in health and dysregulation in psychiatric and autoimmune disorders. Endocrinology and Metabolism Clinics of North America, 23, 451-466.

van der Kooy, K. G., van Hout, H. P., van Marwijk, H. W., de Haan, M., Stehouwer, C. D., \& Beekman, A. T. (2006). Differences in heart rate variability between depressed and non-depressed elderly. International Journal of Geriatric Psychiatry, 21, 147-150.

Wong, M. L., Kling, M. A., Munson, P. J., Listwak, S., Licinio, J., Prolo, P., et al. (2000). Pronounced and sustained central hypernoradrenergic function in major depression with melancholic features: Relation to hypercortisolism and corticotropin-releasing hormone. Procedures of the National Academy of Sciences of the United States of America, 97, 325-30.

Zanstra, Y. J., Schellenkens, J. M. H., Schaap, C., \& Kooistra, L. (2006). Vagal and sympathetic activity in burnouts during a mentally demanding workday. Psychosomatic Medicine, 68, 583-590. 